\title{
Aprendizaje práctico de las propiedades hidráulicas de los materiales geológicos
}

\author{
Practical learning OF the hydRaUlic PROPERTIES OF GEOLOGICAL Materials \\ José SelLÉs-Martínez \\ Departamento de Ciencias Geológicas, Facultad de Clências Exatas y Naturales, Universidad de Buenos Aires, Buenos Aires, Argentina. \\ E-MAIL: PEPE@GL.FCEN.UBA.AR
}

\begin{abstract}
The fight against the popular belief about the widespread presence of underground rivers and lakes is addressed by formal education through the early introduction of the aquifer concept. Knowledge of the hydraulic parameters (porosity, permeability, etc.) is necessary for the correct understanding of the behavior of geological materials as such water reservoirs. At this stage, in addition to adequate basic information, an experimental visualization of what these parameters represent is necessary to achieve a full understanding of the subject. Generally, when experiments are carried out at school, measurements are made with different devices and materials and in a non-integrated way, what works against the construction of an integrated conceptual model by the student. A simple activity (and inexpensive in terms of the necessary equipment) is proposed so that students can perceive how the hydraulic parameters are linked to each other and observe the hydraulic behavior of different geological materials.
\end{abstract}

Resumo: La creencia popular acerca de la existencia universal de ríos y lagos subterráneos es enfrentada desde la educación formal mediante la introducción temprana del concepto de acuífero. El conocimiento de los parámetros hidráulicos (porosidad, permeabilidad, etc.) es necesario para la comprensión del comportamiento de los materiales geológicos como tales reservorios de agua. En esta etapa, además de información básica adecuada, es necesaria una visualización experimental de lo que estos parámetros representan para lograr una comprensión cabal del tema, Generalmente, cuando se realizan experiencias, las mediciones se realizan con aparatos y materiales diferentes y en forma no integrada, lo que atenta contra la construcción de un modelo conceptual integrado por parte del alumno. Se propone una actividad sencilla (y económica en cuanto al equipamiento necesario) para que los alumnos puedan percibir cómo los parámetros hidráulicos se vinculan entre sí y observar el comportamiento hidráulico de diferentes materiales geológicos.
Citation/Citação: Sellés-Martínez, J. (2021). Aprendizaje práctico de las propiedades hidráulicas de los materiales geológicos. Terræ Didatica, 17(Publ. Contínua), 1-9, e021004. doi: 10.20396/td.v17i00.8663891.

Keywords: Hydrogeology, Infiltration, Porosity, Permeability, Transmissivity, Aquifer.

Palabras clave: Hidrogeología, Infiltración, Porosidad, Permeabilidad, Trasmisividad, Acuífero.

Manuscript/Manuscrito:

Received/Recebido: 09/01/2021

Revised/Corrigido: 20/01/2021

Accepted/Aceito: 01/02/2021

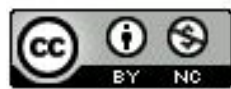

\section{Introducción}

En los últimos tiempos, el tratamiento integrado de los problemas del Medio Ambiente se ha constituido en el modo de abordaje de muchos conocimientos de las Ciencias de la Tierra, enfocándoselos desde una perspectiva más socializada del conocimiento científico. En este aspecto, al ser el agua un recurso vital cuyas mayores reservas se encuentran en el subsuelo, es de fundamental importancia que la sociedad se haga cargo de su defensa y preservación. Tal como señalan Castilho-Barbosa et al. (2020):

"Consolidar la protección, conservación y uso sustentable del recurso requiere la construcción de un sentido de apropiación por parte de la comunidad y expandir un cierto sentido de responsabilidad con respecto a la preservación del patrimonio agua subterránea" (Castilho-Barbosa et al., 1920, p.1).
Para lograr esa apropiación y ese sentido de responsabilidad se hace imprescindible la posesión, por parte de la sociedad, de una acabada comprensión del funcionamiento de los sistemas acuíferos, para poder entender cabalmente cuáles son sus fortalezas y debilidades. Entre las muchas propuestas de educación geo-hidrológica existentes pueden mencionarse López Geta et al. (2001), Ben-Zvi Azaraf et al. (2007), Meléndez Hevia (2009) y diversos materiales didácticos producidos por el Instituto Geominero de España, así como también las propuestas de actividades desarrolladas por la iniciativa Earth Learning Idea (King et al. 2009). Sin embargo es importante prestar atención, ya que en muchos casos, las empresas vinculadas a la extracción, tratamiento y comercialización del agua han producido materiales para divulgación que a veces contienen errores derivados de la sobresimplificación o, incluso, del desconocimiento acerca de la hidrogeología subterránea. En otros 
casos (por fortuna cada vez menos frecuentes) los componentes subterráneos del Ciclo del Agua son considerados con bastante menor énfasis que los atmosféricos y superficiales. Esta subestimación se contradice con las menciones que, en otras situaciones y ámbitos, se hacen acerca de que las aguas subterráneas son sumamente importantes y que todos aquéllos factores que condicionan su infiltración, almacenamiento y circulación tienen una importancia fundamental a la hora de comprender la relevancia de las reservas subterráneas y los problemas que su contaminación puede ocasionar (de Miguel et al. 2009, Márquez \& Bach, 2007, Reyero et al. 2007).

En este contexto, la comprensión de las propiedades hidráulicas de los materiales geológicos (tanto sedimentos como rocas) es de fundamental importancia y estos temas están siendo incorporados paulatinamente a los textos y artículos de divulgación. A pesar de ello, cuando se realizan experiencias de aula, la mayoría de las veces los parámetros hidrogeológicos son tratados por separado y su medición (en aras de la obtención de un valor lo más exacto posible) se realiza utilizando aparatos específicos que en muchos casos funcionan como "cajas negras". Esta práctica atenta contra la comprensión de que las propiedades hidráulicas de un material están relacionadas entre sí y, como consecuencia natural, atenta también contra la comprensión del modo en que lo están. En la presente contribución se sugiere una aproximación menos exacta de la medición en aras de obtener una mejor visualización de lo que ocurre y una comprensión más relevante de su significado conceptual y de las interrelaciones implícitas.

\section{Fundamentos conceptuales}

Se reseñan a continuación algunos puntos de interés para la comprensión de los objetivos y metodología de la propuesta.

\section{Saber cómo se almacena el agua en el subsuelo}

¿Dónde se encuentra el agua en el subsuelo? ¿Cómo llega allí? ¿Desde dónde? ¿Se mueve? ¿Cómo? ¿Hacia dónde? ¿Por qué? Todas estas preguntas están directamente ligadas a una serie de conceptos geológicos, hidrogeológicos e hidráulicos y sus respuestas no siempre son conocidas por el público en general y los alumnos (véanse, por ejemplo, los estudios llevados a cabo por Ben-zvi-Assarf \& Orion, 2005, Dickenson et al. 2005, Márquez \& Bach 2007, Reyero et al. 2009). Más allá de la información que pueda proporcionarse al respecto diciendo que el agua (y los fluidos en general) se encuentran ocupando espacios vacíos (poros y fracturas), es necesaria una correcta comprensión de los parámetros físicos que controlan la infiltración, almacenamiento y circulación del agua en el subsuelo. Esta comprensión, como ya se ha dicho, es imprescindible para desarrollar una cabal conciencia de la importancia de la explotación racional del recurso y de la preservación de la cantidad y calidad del agua subterránea y lo es también para la toma de las decisiones en una sociedad correctamente informada cuya opinión en las redes sociales e incluso su voto, pueden influir en las políticas de explotación de los recursos y en la conservación de la calidad del Medio Ambiente.

La idea de que el planeta Tierra debería llamarse "Planeta Agua", ya que la misma ocupa el $70 \%$ de su superficie, dejándose de lado que no se trata de un problema de superficies sino de volúmenes, se encuentra sumamente extendida y suele aparecer en algunos libros de texto. Esta idea no toma en consideración que el espesor de esa capa es casi despreciable frente al radio terrestre y que, en consecuencia el agua es mucho más escasa de lo que la expresión "70\%" sugiere. De modo similar, pensar que las aguas subterráneas ocupan gigantescos embalses subterráneos al mejor estilo de los hidrofiláceos del "Mundus Subterraneus" de Atanasius Kirchner o las cavernas del "Viaje al centro de la Tierra" de Julio Verne y están conectadas por ríos subterráneos, induce a pensar en velocidades de circulación muy altas y volúmenes limitados a la magnitud de las cavernas y los canales que las unen. La realidad es diferente, si bien la velocidad de circulación del agua en el subsuelo es mucho menor que la del agua que corre por un canal o tubo, el volumen de agua allí almacenada es, de modo contra-intuitivo, mucho mayor del que podría almacenarse en cavernas subterráneas sin que se generaran problemas de colapso. Nunca se repetirá demasiadas veces que los fenómenos kársticos (a los que los conceptos populares acerca del agua subterránea están más ligados), son más espectaculares que habituales y ellos están muy condicionadas tanto por el clima, como por la litología de las rocas, los procesos de ascenso y descenso de los bloques regionales y la evolución geomorfológica de la región. 


\section{¿Experimentar? ¿Con qué objetivo?}

En muchos casos los docentes hacen excesivo hincapié en el tratamiento teórico y en el planteo casi exclusivamente matemático de los temas vinculados a la ciencia, descuidando la potencialidad del aprendizaje de los conceptos por observación y experimentación y ejercitando la deducción. En las etapas de aprendizaje pre-universitario de las ciencias es tan relevante aprender los conceptos científicos como las actitudes y metodologías que hacen al desarrollo de las diferentes disciplinas. La posibilidad de interactuar con los materiales, planificar experiencias, realizarlas, predecir lo que podría ocurrir, llevar un registro de las mediciones, etc. y, finalmente, analizar los resultados obtenidos, tiene un valor pedagógico que supera al del aprendizaje meramente formal de las definiciones y fórmulas, que sólo son signos que representan (textual y matemáticamente) las propiedades en cuestión y que carecen de valor cuando sólo son aprendidas de modo memorístico y sin vinculación con la "circunstancia" de quién aprende.

Buenos ejemplos de experimentación acerca de los parámetros hidrogeológicos en el aula pueden encontrarse en Passey et al. (2006) y Nicholl \& Scott (2000). Es por ello que en esta propuesta se ha hecho especial énfasis en los modos de proceder, las preguntas que preceden a las mediciones y en la realización de descripciones (gráficas y textuales) de lo que se observa (tanto elementos como fenómenos) como un modo de "entrenar" al estudiante en los procesos de "construcción" de conocimiento, aún cuando no sea la intención de la propuesta explorar en profundidad técnicas de aprendizaje por descubrimiento. Esta actividad puede ser un adecuado paso previo a otras más complejas (como las sugeridas por Warner \& Roof, 1994; Gates et al. 1996; Rich \& Onasch 1997; Castaño et al. 2008; King et al. 2009) en las que se investigan con mayor profundidad y precisión las propiedades hidrogeológicas de los materiales y para las cuales es necesaria la comprensión del significado de dichos parámetros.

\section{Ver para aprender. ¿Cómo se almacena y circula el agua en el subsuelo?}

La propuesta se presenta y desarrolla de acuerdo a un criterio de organización que ha sido considerado adecuado por el autor. Sin embargo, se trata de una entre varias posibilidades y puede ser reorganizada y modificada de acuerdo a las circunstancias o intereses particulares de cada docente.

\section{Lo hacemos entre todos}

La actividad es encarada como un trabajo en equipo, en el cual el docente organiza, propone y supervisa, pero son los alumnos quienes se ocupan de la realización de las tareas. Esto es de importancia para el desarrollo de las habilidades para planificar el hacer y llevarlo a cabo, así como también para aprender a coordinar diversas tareas que deben ser realizadas en forma simultánea o secuencial por parte del grupo. Este tipo de habilidades son consideradas fundamentales en el aprendizaje de la ciencia que, es importante insistir en ello, no debe limitarse exclusivamente a los conceptos sino extenderse a los modos de hacer. Es en esta misma línea que permanentemente se solicita, a lo largo de la propuesta, la realización de descripciones y la confección de dibujos (frecuentemente a diferentes escalas de observación) como una manera de contribuir al desarrollo de las capacidades para observar y describir (tanto en forma textual como gráfica). El docente deberá tener en cuenta en estos casos que el propósito de dibujar no es la obtención de una ilustración artística o la búsqueda de la perfección fotográfica, sino que el alumno habitúe y entrene su vista para "recorrer" los materiales y reconocer sus características. Es por ello que se descarta la toma de fotografías, salvo que, a posteriori, se desee elaborar un informe y documentar las actividades realizadas.

\section{¿Qué se espera de la actividad?}

La propuesta que se desarrolla en los apartados siguientes permitirá:

1. Reconocer la necesidad de contar con espacios vacíos para almacenar y trasmitir agua

2. Establecer la relación entre los espacios vacíos (porosidad) y el volumen total de la muestra

3. Determinar la importancia del grado de conexión entre dichos espacios para ampliar o disminuir el potencial para almacenar agua (porosidad efectiva)

4. Poner en evidencia la paradoja existente entre el tamaño de los poros y la cantidad total de agua que puede almacenar el material

5. Determinar la importancia del tamaño de los poros en la velocidad de drenaje del material

6. Observar que no toda el agua contenida en un material es drenada cuando se deja escurrir la misma bajo la influencia de la gravedad

\begin{tabular}{c|c|c|c|c|c}
\hline (C) Terrae Didat. & Campinas, SP & v.17 & $1-9$ & $\mathrm{e} 021004$ & 2021 \\
\hline
\end{tabular}


El modo en que se hilvanan los diferentes pasos de la experiencia permitirá crear un esquema mental de cómo se relacionan unas propiedades con otras y con las características físicas (forma, tamaño y homogeneidad) de las partículas que componen los sedimentos y las rocas.

\section{Los parámetros que serán considerados}

Los parámetros hidrogeológicos que se tomarán en consideración en la estructuración de la experiencia son:

- porosidad

- permeabilidad

- retención

- se mencionarán además la importancia de, y las diferencias entre, densidad y viscosidad

Estos parámetros son parte fundamental de la expresión de las leyes que rigen el comportamiento del agua en el subsuelo y, por lo tanto, su adecuada comprensión es la llave para la comprensión del significado de las mismas. Otros parámetros hidrogeológicos, como el coeficiente de almacenamiento, si bien de gran importancia (sobre todo en el caso de acuíferos confinados) resultan de más difícil visualización y por lo tanto no serán considerado en este nivel de aproximación, del mismo modo que tampoco lo serán los problemas de reactividad química entre el medio y el agua.

\section{Metáforas y analogías en los títulos de las secciones}

En algunas de las secciones que van describiendo las actividades se han utilizado títulos que podrían parecer "poco académicos". Sin embargo esos títulos, coloquiales si se quiere, contienen referencias claras a los materiales y las propiedades que se van a investigar en cada uno de los puntos. Una discusión con los alumnos, sobre todo los más pequeños, acerca de los títulos y de qué se quiso señalar en ellos puede enriquecer la experiencia, brindando un escenario diferente para el análisis de la comprensión lograda, en función de la capacidad de relación entre los conceptos científicos y las metáforas y analogías utilizadas.

\section{Materiales necesarios}

Se utilizarán materiales naturales (rocas ígneas, metamórficas y sedimentarias y también sedimen-

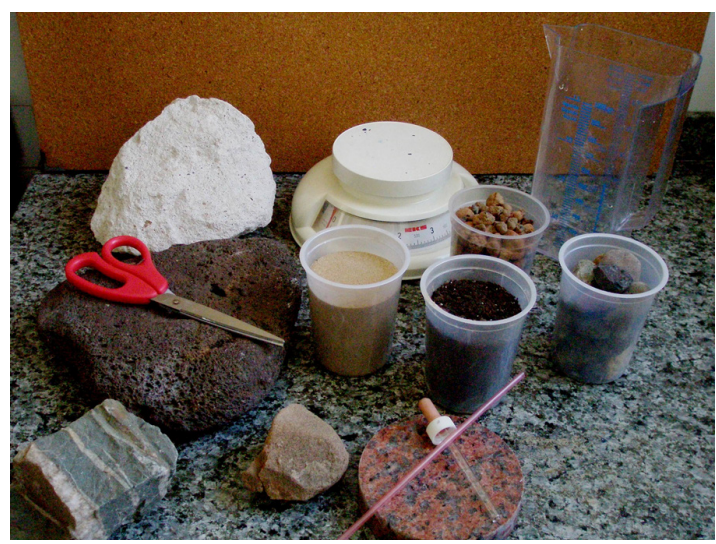

Figura 1. Materiales necesarios para la realización de la experiencia

tos) y algunos materiales de origen industrial análogos de éstos (plásticos o vidrios impermeables, ladrillos, tejas, etc.). Se necesitarán vasos plásticos transparentes de tipo descartable (o algún recipiente similar), una jarra graduada para adicionar agua a los recipientes señalados, una balanza de cocina para pesar los mismos, un gotero, aceite, plastilina y pajitas, que se ilustran en la figura 1.

\section{Descripción de las actividades}

\section{1- La materia es impenetrable: el agua sólo ocupa lugares vacíos}

1a. Este punto se ilustra comparando la posibilidad de humedecerse de dos materiales diferentes de presencia común en la naturaleza: una muestra de roca cristalina (preferentemente ígnea) y otra de arcilita o limolita (Fig. 2). En este caso puede realizarse una modelización analógica utilizando un trozo de vidrio para representar la primera y uno de ladrillo o teja para el caso de la segunda. Es todavía mejor si se duplica la experiencia con los materiales naturales y sus análogos y se discute acerca de las posibles comparaciones entre ambos (en qué se parecen y en qué se diferencian y las implicancias de ello). Se pide a los alumnos que realicen un pequeño diagrama del aspecto de cada una de las muestras y que anticipen qué ocurrirá si, con la ayuda de un gotero se deja caer una gota de agua sobre los mismos, solicitándoles que justifiquen sus respuestas.

Una vez realizada la experiencia, se discuten los resultados en relación con las expectativas y se les proporciona una lupa o lente de aumento potente para que observen con mayor detalle 
la superficie de cada muestra. Se les pide que nuevamente, y a esta escala ampliada de observación, describan y dibujen lo que ven, debiendo incorporarse obviamente al diseño la presencia, en las rocas sedimentarias y sus homólogos, de los espacios porales, seguramente ausentes en el dibujo realizado antes de mojar las muestras. Puede mencionarse que, las gotas que han escurrido del gotero, son equivalentes a las gotas de la lluvia que caen sobre la superficie terrestre. Resbalan sobre las superficies impermeables pero empapan las rocas porosas y los sedimentos. El proceso por el cual las gotas pasan de la superficie al interior del material se denomina infiltración.

1a. En una segunda parte de la experiencia se pesan sendas muestras de una roca cristalina y de otra sedimentaria (ambas secas), se sumergen luego en agua y vuelven a pesarse. Se pregunta cuál es la razón por la cual el fragmento de roca sedimentaria pesa más luego de haber sido sumergido que cuando estaba seco y se les propone que encuentren el significado de la diferencia de peso determinada. Se pretende así que, con la ayuda del docente, lleguen a la expresión que relaciona la diferencia de peso con el volumen de espacios huecos existentes en el material y que pueden ser ocupados por el agua, es decir con su porosidad efectiva. El desprendimiento de burbujas (Fig. 3 ) es un claro indicio de que el aire almacenado en los poros está siendo desplazado por el agua que penetra en el material.

\section{2- Las apariencias engañan. Una gran cantidad de agujeros no asegura que quepa mucha agua}

El punto que surge aquí es el hecho, no intuitivo para los alumnos, que los materiales geológicos pueden contener poros aislados, en los que no puede penetrar el agua, tema que se aborda en el próximo paso. Para esta experiencia hacen falta dos muestras "engañosas": una de ellas un basalto con grandes y abundantes vesículas y la otra un material arenoso cohesivo, de poros pequeños pero muy interconectados (Figs. 4 y 5). Se pide a los alumnos que describan ambas muestras y anticipen cuál de ellas será capaz de almacenar más agua. Las muestras se sumergen en forma consecutiva en sendos recipientes con agua y en cada caso se describe lo que ocurre (¿Se desprenden burbujas? ¿Muchas? ¿El proceso persiste en el tiempo o se detiene rápidamente?).

Se pide luego a los alumnos que elaboren una explicación para lo observado y las razones por las

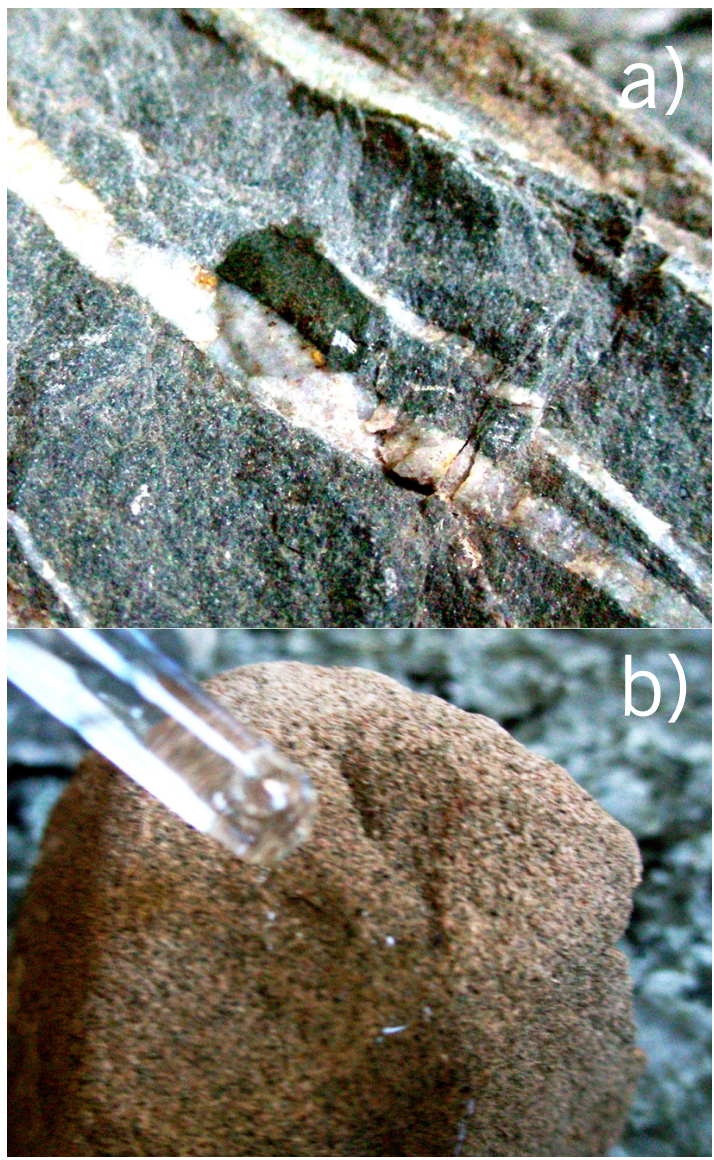

Figura 2. a) La impermeabilidad de la roca ígnea impide que el agua penetre en la misma, la gota permanece en la superficie de ella. b) La permeabilidad de la roca sedimentaria permite que el agua empape el material, que toma un color más oscuro

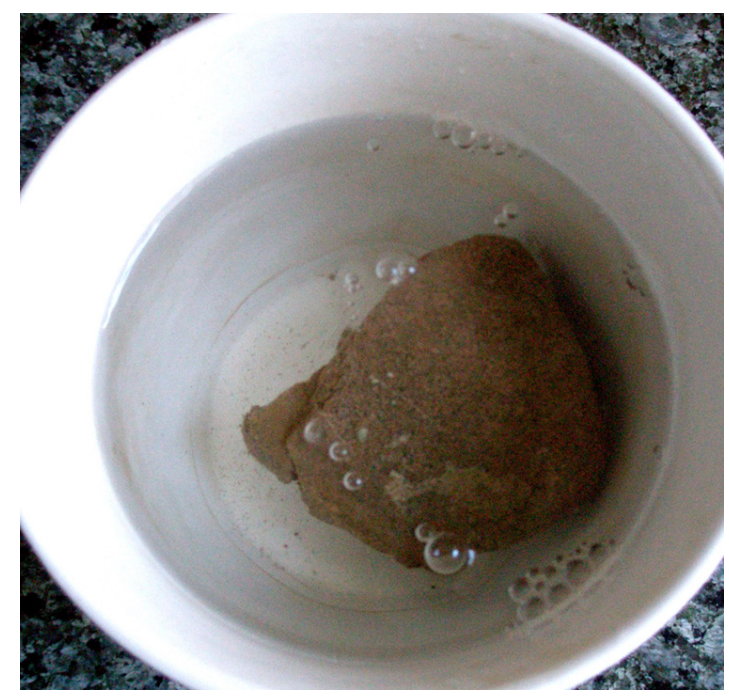

Figura 3. Burbujas desprendiéndose de un trozo de roca sedimentaria sumergida en agua

\begin{tabular}{c|c|c|c|c|c|}
\hline (C) Terrae Didat. & Campinas, SP & v.17 & $1-9$ & $\mathrm{e} 021004$ & 2021 \\
\hline \multicolumn{2}{|c|}{5}
\end{tabular}




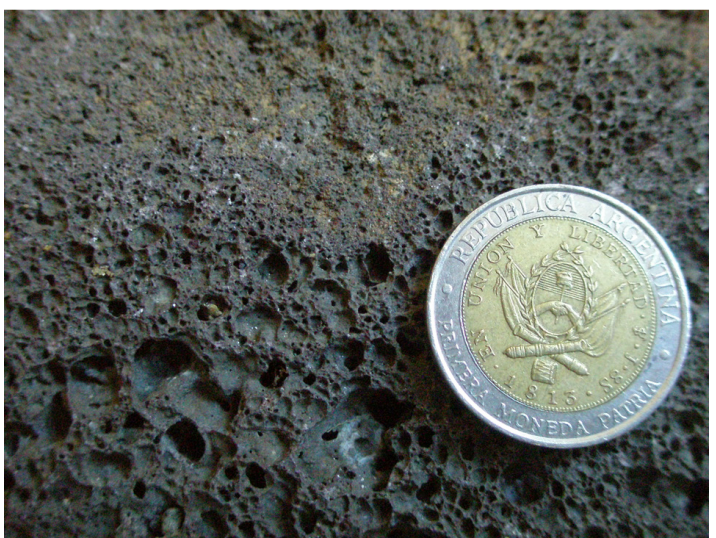

Figura 4. Textura del basalto. Pueden observarse las cavidades correspondientes a las burbujas formadas en la lava antes de su enfriamiento. Estas cavidades no están conectadas entre sí, por lo que el agua no puede circular a través de ellas

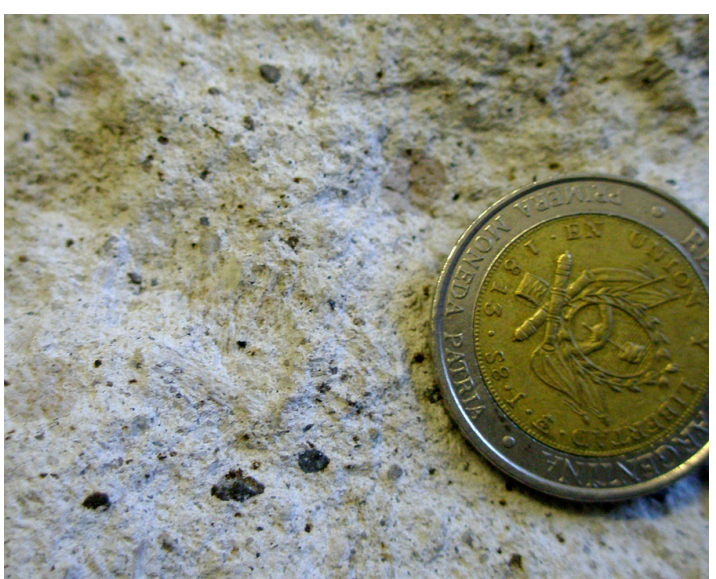

Figura 5. Textura de un depósito clástico. Pueden observarse algunos clastos de tamaño mayor y color más oscuro. El tamaño de los poros no es visible a ojo desnudo, sin embargo, al estar los mismo conectados entre sí permiten el ingreso y la circulación del agua en la muestra

cuales sus pronósticos coincidieron o no con los resultados previstos. Con la ayuda de la lupa o lente de aumento deben describir y dibujar en detalle la superficie de ambos materiales y vincular esta observación minuciosa con el grado de conexión o no entre los poros y cavidades.

Se ha puesto así de relieve el hecho que no basta con que haya espacios huecos, sino que es necesario que los mismos estén intercomunicados para que el agua pueda circular y ocuparlos. Una vez que todos tienen claro que el agua sólo ha ocupado los espacios interconectados, puede desarrollarse la fórmula matemática que expresa el valor de la porosidad efectiva:

$$
\text { Porosidad Efectiva }=\mathrm{V}_{\text {espacios conectados }} / \mathrm{V}_{\text {total }}
$$

Una segunda pregunta, que debería disparar una serie de observaciones acerca de la complejidad de la determinación de la porosidad total que incluye a los espacios que no están conectados entre sí, es justamente pedirles que diseñen un método para medirla. Dicho método involucra cálculos y mediciones de mayor complejidad, pues se hace necesario conocer el volumen aparente del material (determinado por la forma y tamaño de la muestra) y el volumen real (espacio que ocuparía si no tuviera poros) y para ello debe incorporarse al cálculo la densidad del material. Avanzar más o menos en este planteo queda a criterio de los docentes y está supeditado al conocimiento que los alumnos posean de Física y Matemáticas.

\section{3- ¿Dónde cabe más agua? iEn pocos agujeros grandes 0 en muchos pequeños?}

La obtención de resultados paradójicos suele ser un método adecuado para la incorporación de conceptos nuevos y es por ello que se propone la incorporación de esta actividad en este punto. Contrariamente a lo esperado por "el sentido común", no es en un material con grandes espacios vacíos aquél en el cual lograremos almacenar más agua, sino uno en el cual los poros interconectados, aunque apenas perceptibles al ojo desnudo, forman una red muy densa. Para la realización de esta parte de la experiencia son necesarios dos recipientes llenos hasta su borde, uno con gruesos fragmentos de roca cristalina (de $1 \mathrm{~cm}$ a $2 \mathrm{~cm}$ de diámetro aproximadamente) y el otro con arena gruesa. Se pregunta a los alumnos cuál de ellos será el que almacene más agua y porqué. Se pesan ambos recipientes con los materiales secos y luego se vierte agua en cada uno de ellos hasta que la misma cubre completamente al material. Se procede a pesarlos y se discuten los resultados obtenidos. Cuanto mayor sea el tamaño del recipiente utilizado mayor será la diferencia en los volúmenes y menor la influencia del contacto de los materiales con el borde del recipiente y, por lo tanto, más fácil de apreciar la variación en la medición.

\section{4- Mezclados cabemos más... y le dejamos menos lugar al agua}

En este paso de la experiencia se compara la influencia de la selección (u homogeneidad en el 
tamaño de grano) en la porosidad efectiva de un material. Para ello se toman materiales de diferente tamaño de grano (gravas, arenas gruesas, arenas finas) y se colocan en diferentes recipientes, llenándolos hasta el borde. Un recipiente conteniendo una mezcla de todos ellos se prepara por separado. Los recipientes deben tener la posibilidad de ser drenados con facilidad y sin pérdida del material sólido ya que será necesario hacerlo en un próximo paso de la experiencia. En la Fig. 6 se ilustra una construcción económica y adecuada a los fines propuestos. Un broche de apretar papeles puede servir perfectamente para apretar la pajita y abrir o cerrar el flujo de líquido a través de ella.

Una vez preparados los recipientes se numeran con tinta indeleble, se pesan en estado seco y las mediciones son recogidas en una tabla. Una vez realizado el pesaje se vierte agua en cada uno de ellos hasta su borde. Como en los casos anteriores, los alumnos deben anticipar cuál podrá almacenar más agua y porqué. La cantidad de agua puede medirse tanto por diferencia de volumen en la jarra graduada que se utiliza para verter el líquido como por diferencia de peso en cada recipiente. Se anotan las mediciones en una tabla, se discuten los resultados obtenidos y se justifican las coincidencias y disidencias con los pronósticos realizados.

\section{5- Una carrera con obstáculos en la que el resultado puede depender de las vallas... 0 del corredor}

En los apartados anteriores se han explorado las propiedades hidrogeológicas que podríamos llamar "estáticas" y en las cuales no se han tenido en cuenta el tiempo que le ha llevado al agua "empapar" o drenar completamente cada material. En los próximos pasos se explorarán aquéllas propiedades que controlan el desplazamiento del agua, facilitándolo o retardándolo e influyendo, por lo tanto, en la velocidad con la que el agua circula y en la cantidad de agua que puede obtenerse en un determinado plazo (según sea la perspectiva con que se encare la experiencia). Aparece aquí el concepto de permeabilidad y de su relación tanto con la cantidad, geometría y conectividad de los poros como con las características del material que los atraviesa. Para ello se realizarán dos series de experiencias utilizando dos baterías iguales de tres recipientes con materiales de distinto tamaño de partícula. En la primera de ellas se comparará el comportamiento del agua al atravesar diferentes

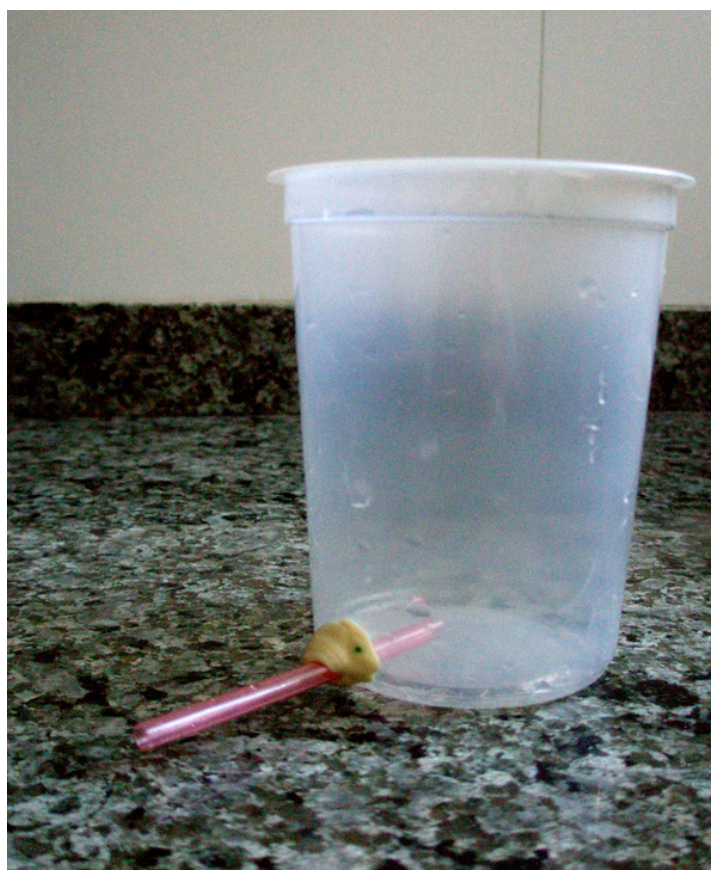

Figura 6. Recipiente preparado para un fácil drenaje, para ello se pasa un trozo de pajita plástica por un pequeño agujero y se sella el borde con plastilina. La salida de agua puede habilitarse o cerrarse con un broche para papeles (no visible en la fotografía)

materiales y en la segunda se empleará aceite en lugar de agua.

5a. Se llenan los recipientes de la primera batería con agua hasta saturar el material. Luego debe realizarse la medición del tiempo que tarda en pasar por el sistema una cantidad adicional de agua que debe ser la misma en los tres casos (por ejemplo 100 mililitros). Este paso debe realizarse con paciencia para que el agua no desborde, pero tratando de no interrumpir el flujo de agua que se está agregando. Para que la experiencia se realice adecuadamente es necesario que, al comenzar a verter el agua adicional, se abra la parte inferior del recipiente y se deje circular libremente el líquido, recogiéndolo en otro recipiente o, simplemente, dejándolo caer en una pileta.

$5 b$. Luego se procede en forma similar con la segunda batería de recipientes, pero utilizando aceite en lugar de agua. Se realiza primero la saturación y luego la medición del tiempo de circulación. Se comparan los tiempos en cada caso, tanto en forma general como de a pares por recipientes rellenos con la misma granometría. Los tiempos requeridos ¿Son siempre los mismos? ¿Se hacen mayores para granometrías más pequeñas? ¿Qué diferencias se observan entre los tiempos requeridos por el agua y por el aceite?

\begin{tabular}{c|c|c|c|c|c}
\hline (C) Terrae Didat. & Campinas, SP & v.17 & $1-9$ & $\mathrm{e} 021004$ & 2021 \\
\hline
\end{tabular}


Es esta, además, una buena oportunidad para ayudar a los alumnos a diferenciar los conceptos de densidad y viscosidad. Esto no es trivial, ya que muchas veces, en la lengua cotidiana se hacen sinónimos densidad y viscosidad y, aún sin llegar a ese extremo, en general se asocia una mayor viscosidad con un material más denso. El caso del agua y el aceite demuestra claramente que ambas propiedades no están rígidamente ligadas. El aceite es más viscoso que el agua, pero flota sobre ella porque es menos denso y, a pesar de ser más liviano, le cuesta más circular por los poros. Una técnica adecuada para la medición de la permeabilidad de trozos irregulares de roca ha sido descripto por Hudak (2001), recomendándose su exploración si se desea añadir algo de complejidad a esta experiencia.

\section{No toda el agua que vertimos en cada recipiente se recupera después}

La medición de la permeabilidad nos permite además comprobar otro fenómeno interesante: la adherencia del agua a la superficie de los materiales y su resistencia a abandonar los poros una vez que los ha ocupado (para una explicación sencilla pero correcta de esta propiedad puede verse Davis \& Day (1971). Al permitir el escurrimiento por gravedad de cada uno de los recipientes, puede verse que no se recupera toda el agua (o aceite) vertidos en cada uno de ellos (el material no queda "seco"). La fracción faltante es función tanto de las propiedades del material (composición química y tamaño y forma de los poros) como del fluido. El parámetro que describe y cuantifica esta propiedad se denomina retención del material. Para la medición de este parámetro pueden aprovecharse los datos correspondientes a las baterías de recipientes ensayadas en el punto precedente, dejando escurrir completamente el líquido contenido en ellos y agregando este nuevo valor como una columna más a la tabla. Nuevamente pueden compararse las retenciones correspondientes a cada fluido según la granometría que atraviesa y de cada granometría con respecto al fluido ensayado.

\section{Conectar las observaciones y mediciones con la realidad y el entorno}

Es imprescindible, cuando se habla de la enseñanza de temas de ciencias básicas, realizar un especial hincapié en la necesidad de puntualizar las relaciones entre las observaciones y mediciones que se realizan en el aula-laboratorio con lo que se ve y ocurre en la naturaleza, sobre todo en el entorno de los estudiantes. Experiencias como la ya señalada de "El Planeta Azul" (Ben-Zvi Assaraf et al. 2007) evidencian claramente que las posibilidades de interesar a los alumnos en los conceptos básicos y lograr aprendizajes significativos se multiplican cuando los alumnos advierten que dichos conceptos les permiten comprender los problemas de la vida y entorno cotidianos. Es por ello que antes o después (y organizado según corresponda en cada caso) debe planificarse alguna salida al medio exterior para que los alumnos capten o apliquen (según corresponda) las relaciones entre los fenómenos naturales y las experiencias de laboratorio. Aún en ambientes urbanos pueden encontrarse modos de realizar esta vinculación. A modo de ejemplo podemos mencionar que la experiencia descripta en el primer paso de nuestra propuesta, la comparación entre la capacidad para absorber agua de una roca cristalina y un material arcilloso es un análogo perfecto del problema de la incapacidad de los suelos y cubiertas impermeables para absorber el agua de la lluvia y, por lo tanto, la causa de las inundaciones cuando la "porosidad artificial" provista por la red pluvial de la ciudad se ve superada por el volumen de agua caída y la capacidad de la red de drenar el exceso de agua a la misma velocidad con que la lluvia la provee. Comparar lo que ocurre cuando, siempre dentro de la ciudad, la lluvia cae sobre los árboles y sobre el césped de un parque y cuando lo hace sobre el asfalto de la calle puede dar motivo a reflexiones muy valiosas acerca de aspectos como la infiltración, la escorrentía superficial y la acción erosiva del agua.

\section{Consideraciones finales}

Se han desarrollado en los párrafos precedentes una serie de actividades sugeridas con el propósito de contribuir a alcanzar un aprendizaje significativo, por parte de los alumnos, de los conceptos vinculados a la infiltración, almacenamiento y la circulación del agua en el subsuelo. Estas actividades deberán ser acompañadas, sin duda, por acciones complementarias como el desarrollo teórico de los temas, la presentación de casos ejemplos de acuíferos locales y regionales y la posibilidad de que los alumnos realicen algunos trabajos de investigación escolar asociados a problemas hidrogeológicos en el área en que viven. La realización de actividades de campo, como por ejemplo, un recorrido por una 
zona en la cual afloren rocas ígneas o metamórficas, rocas sedimentarias, depósitos de sedimentos y se desarrollen suelos, puede ser una ocasión magnífica para que los estudiantes recojan, bajo la supervisión del docente, las muestras que luego serán ensayadas de acuerdo a la rutina sugerida.

Como siempre, es la creatividad del docente la que, a partir de la "receta pedagógica" ofrecida, puede llevar el tema por los caminos más adecuados a los conocimientos y capacidades de sus alumnos y lograr los mejores resultados.

Dígase para finalizar que el paso siguiente en la comprensión del funcionamiento de los materiales geológicos que almacenan agua y constituyen acuíferos es el abordaje de la denominada Ley de Darcy, que permite cuantificar el caudal de agua que circulará por una determinada sección del material en función tanto de sus propiedades hidráulicas como de la diferencia de presión entre el punto de ingreso y salida del fluido. El desarrollo de la experiencia y su importancia como ejemplo del modo de construcción del conocimiento científico y de la transformación de ideas y conceptos en una fórmula, es de suficiente interés como para justificar su tratamiento por separado, el que será motivo de una próxima contribución del autor.

\section{Agradecimientos}

El autor agradece al Celso Dal Ré Carneiro, a Gerôncio Rocha y a otro revisor anónimo sus amables comentarios y sugerencias.

\section{Referencias}

Ben-Zvi Assaraf, O. \& Orion, N. (2005). A study of junior high students' perception of the water cycle. Journal of Geoscience Education, 53(4), 366-373. doi: 10.5408/1089-9995-53.4.366.

Ben-Zvi Assaraf, O., Orion, N., Ben Menajem, O., Yardén, A., Ronen, D. \& Lemcoff, J. H. (2007). El Planeta Azul, el Ciclo del Agua en los Sistemas Terrestres. Instituto Weizman. Israel. 262p.

Castaño, S., Moreno, L., de la Losa, A., Aguilera, H., Jiménez, M. E. \& Mediavilla, R. (2008). Evaluación de pérdidas de agua y parámetros hidráulicos mediante ensayos de infiltración en campo. Enseñanza de las Ciencias de la Tierra. 16(1), 64-71. URL: https:// www.raco.cat/index.php/ECT/article/view/120988. Acesso 6.01.2021.

Castilho-Barbosa, I. N. B., Carvalho, A. M., Miguel, G. F. \& Carneiro, C. D. R. (2020). Conhecer para conservar aquíferos: como torna-los visíveis? $\mathrm{Te}$ rre Didatica, 16(1), 1-12, e020018. doi: 10.20396/ td.v16i0.8658299.

Davis, K. S. \& Day, J. A. 1971. Agua, espejo de la ciencia. EUDEBA, Buenos Aires, 218 p. de Miguel, A., Lado, J. J., Martínez, V., Leal, M. \& García, R. (2009). El ciclo hidrológico: experiencias prácticas para su comprensión. Enseñanza de las Ciencias de la Tierra, 17(1), 78-85. URL: https:// www.raco.cat/index.php/ECT/article/view/184048. Acesso 6.01.2021.

De Wet, A. P. (1994). Integrating field observations with physical and computer models in an introductory environmental Geology course. Journal of Geoscience Education, 42 (3): 264-272. URL: https://serc.carleton.edu/resources/39515.html. Acesso 6.01.2021.

Dickerson, D., Callahan, T. J., van Sickle, M. \& Hay, G. (2005). Students' conceptions of scale regarding ground water. Journal of Geoscience Education, 54 (4): 487-490. doi: 10.5408/1089-9995-53.4.374.

Gates, A. E., Parker Langford, R., Hodgson, R. M., \& Driscoll, J. J. (1996). Ground water-simulation apparatus for introductory and advanced courses in Environmental Geology. Journal of Geoscience Education, 44(5), 559-564. doi: 10.5408/0022-136844.5.559.

Hudak, P. F., (2001). Creased plastic-rock permeameter for Hydrogeology students. Journal of Geoscience Education, 49 (2), 182-186. doi: 10.5408/10899995-49.2.182.

King, C., Kennett, P., Devon, E. \& Sellés Martínez, J., (2009). Earthlearningidea: nuevos recursos para la Enseñanza de las Ciencias de la Tierra en todo el mundo. Enseñanza de las Ciencias de la Tierra, 17(1), 2-15. URL: https:/www.raco.cat/index.php/ECT/ article/view/183787. Acesso 6.01.2021.

Márquez, C. \& Bach, J. (2007). Una propuesta de análisis de las representaciones de los alumnos sobre el ciclo del agua Enseñanza de las Ciencias de la Tierra, 15(3), 280-286. URL: https://www.raco.cat/index. php/ECT/article/view/121419. Acesso 7.01.2021.

Meléndez Hevia, I. 2009. La dinámica de sistemas complejos en las ciencias de la tierra y del medio ambiente. Enseñanza de las Ciencias de la Tierra. 17(1), 26-36. URL: https://www.raco.cat/index.php/ ECT/article/view/184043. Acesso 7.01.2021.

Nichol, M. J. \& Scott, G. F. (2000). Teaching Darcy's Law through hands-on experimentation. Journal of Geoscience Education, 48(2), 216-221. doi: 10.5408/1089-9995-48.2.216.

Passey, B. H., T. E. Cerling \& M. A. Chan, (2006). Dam Fun. A Scale Model Classroom experiments for teaching basic concepts in Hydrology and Sedimentary Geology. Journal of Geoscience Education, 54(4), 487-490. doi: 10.5408/1089-9995-54.4.487.

Reyero, C., Calvo, M., Vidal, M. P., García, E. \& Morcillo, J. G. (2007). Las ilustraciones del ciclo del agua en los textos de educación primaria. Enseñanza de las Ciencias de la Tierra, 15(3), 287-294. URL: https://raco.cat/index.php/ECT/article/view/121420. Acesso 7.01.2021.

Rich, C. C. \& C. M. Onasch, (1997). An introductory Geology Laboratory exercise on ground water pollution potential using the DRASTIC System. Journal of Geoscience Education, 45 (5): 404-411. doi: 10.5408/1089-9995-45.5.404.

Warner A. \& S. R. Roof, (1994). Using Darcy Flow Tubes to teach concepts of ground water geology. Journal of Geoscience Education, 42 (3): 220-224. doi: 10.5408/0022-1368-42.3.220

\begin{tabular}{c|c|c|c|c|c|}
\hline C Terrae Didat. & Campinas, SP & v.17 & $1-9$ & $\mathrm{e} 021004$ & 2021 \\
\hline
\end{tabular}

